REOP. Vol. 17, No 1, $1^{\text {er }}$ Semestre, 2006, pp. 75-97

\title{
TENDENCIAS DE LA EDUCACIÓN SUPERIOR EUROPEA E IMPLICACIONES PARA LA ORIENTACIÓN UNIVERSITARIA
}

\author{
TRENDS IN EUROPEAN HIGHER EDUCATION AND IMPLICATIONS \\ FOR STUDENT SUPPORT AND GUIDANCE
}

\author{
Maria J. Vieira* y Javier Vidal** \\ Dpto. Filosofía y Ciencias de la Educación Universidad de León, España
}

\section{RESUMEN}

La Educación Superior se encuentra en un momento de profundos cambios al asumir los retos que implican la sociedad del conocimiento, la globalización, la revolución tecnológica, la diversidad de los estudiantes, el aprendizaje permanente, la rendición de cuentas, entre otros. Especialmente en Europa, estos cambios están liderados por el proceso de construcción del Espacio Europeo de Educación Superior (EEES) en el que se otorga un papel preponderante al aprendizaje de los alumnos. Por tanto, el EEES supone una ocasión excelente para reflexionar sobre el modelo de universidad que queremos construir.

Aprovechando esta excelente ocasión, en este estudio se realiza (1) una revisión de la literatura sobre la situación de la orientación universitaria, (2) una descripción de las tendencias actuales de la Educación Superior y, por último, (3) un análisis de las ventajas e inconvenientes que la actual situación de la Educación Superior suponen para el desarrollo de la orientación universitaria.

Palabras clave: Espacio Europeo de Educación Superior, apoyo al estudiante, servicios de orientación.

\section{ABSTRACT}

Higher Education (HE) confronts an era of increasing change characterised by globalisation, knowledge-based economy, technological revolution, lifelong learning, greater pressures towards accountability, a much more diverse student population, amongst others. Mainly in Europe, the process to-

* María José Vieira es Ayudante de Universidad en el área de Métodos de Investigación y Diagnóstico en Educación del Dpto. de Filosofía y Ciencias de la Educación de la Universidad de León.

** Javier Vidal es Profesor Titular de Universidad en el área de Métodos de Investigación y Diagnóstico en Educación del Dpto. de Filosofía y Ciencias de la Educación de la Universidad de León.

Líneas de Investigación: Estudios sobre Educación Superior. Evaluación de Universidades. Política de Educación Superior. Apoyo al Estudiante e Inserción Laboral de los Graduados.

Este trabajo forma parte de la tesis doctoral titulada Análisis comparado sobre la planificación y organización del sistema de orientación y apoyo al estudiante en las instituciones de educación superior, realizada por María José Vieira y dirigida por Javier Vidal. 
wards the construction of the European Higher Education Area (EHEA), in which the student learning experience is a central issue, is undertaking these changes. In this context, the EHEA is an excellent occasion for rethinking the university.

Considering this situation, this article will (1) review recent literature related to student support and career guidance, (2) describe and discuss the current trends of HE and, (3) analyse the advantages and disadvantages in the relationship and articulation between the current situation of HE and the development of student support and guidance at Higher Education Institutions.

Key words: European Higher Education Area, student support, career guidance services

\section{Introducción}

La Educación Superior, especialmente en Europa, se encuentra en un momento de profundos cambios. Desde finales de la década de los noventa, con la Declaración de Bolonia se consolida y amplía el proceso de construcción del Espacio Europeo de Educación Superior. En este proceso, diferentes países europeos han iniciado la reforma de la estructura de sus enseñanzas universitarias con un cambio de enfoque muy relevante centrado en el aprendizaje de los alumnos. Otro objetivo que se plantea Europa en este proceso es convertirse en lugar de referencia en el ámbito de la formación universitaria para estudiantes de todos los países, velando necesariamente por la garantía de la calidad de la misma. En concreto, las universidades son consideradas como elemento esencial para la consecución del objetivo de convertir a la Unión Europea en la economía basada en el conocimiento más competitiva y dinámica del mundo para el 2010 fijado en el Consejo Europeo de Lisboa de 2000 .

En este contexto de aprendizaje centrado en el alumno, competitividad y calidad de la formación universitaria, la información, orientación y asesoramiento que reciben los estudiantes constituye un elemento fundamental en la reforma de la educación superior.

En este estudio se realiza inicialmente una revisión de los componentes que integran el sistema de orientación y apoyo a los estudiantes universitarios. Posteriormente, se analizan las tendencias actuales de la educación superior, especialmente en Europa para, por último dar respuesta a las ventajas e inconvenientes que estas tendencias suponen para la institucionalización de sistemas de orientación y apoyo al estudiante en las universidades.

\section{La orientación universitaria}

Aunque la definición del término orientación no ha estado exenta de polémicas, parece existir cierto consenso en considerarla como un proceso de ayuda continuo y sistemático dirigido a todas las personas, en todos sus aspectos, poniendo un énfasis especial en la prevención y el desarrollo, que se realiza a lo largo de toda la vida, con la implicación de los diferentes agentes educativos y sociales (Álvarez González y Bisquerra Alzina, 1996: 7). Asimismo, diversos autores coinciden en la delimitación de esta definición en torno a un conjunto de áreas o ámbitos de intervención, modelos o formas de intervención y contextos en los que este proceso tiene lugar (Bisquerra, 1998; Álvarez González y Bisquerra Alzina, 1996; Alvarez Rojo, 1994; Rodríguez Espinar et al., 1993; Rodríguez Moreno, 1995; Jiménez Gámez y Porras Vallejo, 1997; Alonso Tapia, 1995; Vélaz de Medrano, 1998; Sanz Oro, 
2001; Martínez Clares, 2002). En este sentido, la orientación universitaria es el proceso de ayuda dirigido a estudiantes universitarios (aunque se podría incluir también a otros miembros de la comunidad universitaria) en aspectos académicos, profesionales y personales en el contexto de las instituciones de educación superior.

En este estudio partimos de la idea de que la orientación universitaria debe ser entendida como un sistema de orientación y apoyo al estudiante planificado y organizado institucionalmente atendiendo, tanto a las tendencias del sistema de educación superior, como a las peculiaridades de cada institución.

\section{El sistema de orientación como conjunto de funciones}

El sistema de orientación y apoyo al estudiante universitario está formado por un amplio abanico de funciones cuyo objetivo prioritario es facilitar al estudiante su ingreso, posterior estancia en la institución de educación superior e inserción en el mercado laboral mediante el desarrollo de sus capacidades en los ámbitos académico, profesional y personal, independientemente de cómo (qué modelo de intervención), dónde (qué contexto próximo: el aula, residencias de estudiantes, servicios, etc.) y por quién (profesores, tutores, otros compañeros, orientadores o profesionales) sean ofrecidas estas funciones de ayuda.

Con el fin de diferenciar la multitud de funciones que componen el sistema de orientación y apoyo al estudiante universitario, la International Association of Student Affairs and Services (IASAS), asociación internacional preocupada por atender las necesidades de los estudiantes universitarios, junto con la UNESCO, desarrollaron un manual en el que se describen la variedad de actuaciones que las instituciones de educación superior en el ámbito internacional realizan con el fin de ayudar al estudiante (Ludeman, 2001). Los antecedentes de este estudio habría que buscarlos en la Declaración Mundial de la Educación Superior, Educación superior en el siglo XXI: visión y acción (UNESCO, 1998). En esta declaración se incluyeron varios principios directamente relacionados con el sistema de orientación y apoyo a los estudiantes considerando que en un contexto internacional caracterizado por una educación superior con una demanda sin precedentes, por el aumento de la diversificación, y por ser pieza clave del desarrollo económico y sociocultural de las naciones, ésta debería asumir varios retos. Entre ellos, se daba especial énfasis a una educación superior más centrada en el estudiante, considerado éste en su totalidad, mediante la provisión de servicios comprensivos y programas de ayuda integrados en la política de la institución.

Entre este grupo de prestaciones se encuentran servicios o unidades de diversa índole, teniendo en cuenta que en muchos países no se cubren todas las funciones señaladas en esta descripción internacional. Según este estudio, el sistema de apoyo al estudiante está formado por servicios de admisiones, programas de preparación para la universidad, servicios de orientación profesional, académica, psicológica, centros de salud, servicios de atención a estudiantes con discapacidad, asesoramiento financiero, guarderías, capillas o servicios religiosos multiconfesionales - destinados a cubrir las necesidades espirituales de los estudiantes-, asesoramiento para estudiantes adultos, servicios de atención a minorías étnicas, a estudiantes internacionales, gabinetes de orientación sexual, servicios de deportes, de alojamiento, entre otros (Ludeman, 2001). 
Entre la gran variedad de funciones que cubren los servicios y programas que constituyen el sistema de orientación y apoyo al estudiante universitario, es posible realizar una distinción entre aquellas funciones más vinculadas a lo que en el análisis de procesos de las organizaciones se denominan procesos básicos o clave, que en el ámbito de la educación estarían más vinculados al proceso de enseñanza-aprendizaje, que requieren el apoyo de otros procesos de soporte, de carácter administrativo, financiero, de bienestar, de información y de infraestructuras que, extrapolando al contexto de la educación superior, serían aquellos que faciliten el desarrollo de la vida universitaria en general. A pesar de que los procesos básicos son imprescindibles en la organización, es fundamental el cumplimiento de los procesos de soporte ya que, si éstos no funcionan adecuadamente, el cumplimiento de la misión institucional se verá amenazado (Kells, 1997; Rey, 1998; Caselles, 2003).

En esta doble dimensión, diferenciando entre lo básico y lo de soporte, se enmarcan los principales argumentos que permiten justificar la orientación en las instituciones de educación superior. Como elemento básico, la orientación debe potenciar el aprendizaje y el éxito académico proporcionando atención integral al alumno desde la propia docencia, y como elemento de soporte, la orientación favorece la creación de un contexto institucional lo más facilitador posible para unos clientes con unas necesidades concretas, los estudiantes.

En este sentido, la propia concepción que la institución tenga sobre su misión, sus valores y su visión de lo que espera conseguir va a determinar los objetivos relacionados con el sistema de orientación y apoyo al estudiante. Entre los elementos de este sistema, integrado en la misión de la institución, destaca el campus universitario que debe ser considerado como un factor educativo de gran importancia, ya que en el mismo se debe atender a múltiples necesidades de carácter asistencial y de intercomunicación personal y social (SaulnierCazals, 1997; Kuh \& Schuh, 1991).

\section{Propuestas organizativas}

Las propuestas realizadas sobre qué elementos debe incluir el sistema de orientación y apoyo al estudiante y cómo organizarlos varía en función del contexto de educación superior que se considere. A continuación presentamos algunas de las propuestas organizativas más relevantes del sistema de orientación universitario en Estados Unidos y en Europa.

En Estados Unidos, desde principio de los años noventa se ha producido un cambio en el enfoque de la orientación académica en las instituciones de educación superior motivado por los resultados de las investigaciones de Pascarella y Terenzini (1991), Astin (1993), Tinto (1993), entre otros, sobre la influencia de la institución en el desarrollo y el éxito del estudiante universitario (Terenzini, Pascarella, \& Blimling, 1999). Kramer (2000) propone un modelo organizativo de la orientación académica, una parte fundamental del sistema de orientación, partiendo de las diferentes necesidades de los estudiantes en función de su edad, género, procedencia, cargas familiares, etc., considerando las teorías mencionadas sobre la influencia de la institución (ver Ilustración 1). Este modelo se centra en las necesidades individuales de los estudiantes en tres momentos, antes, durante y después de los estudios universitarios. Se debe tener en cuenta que en el sistema de educación superior estadounidense el estudiante cuando ingresa en la institución no ha decidido la titulación que quiere estudiar, no será hasta su tercer año (junior year) cuando toma esa decisión. 


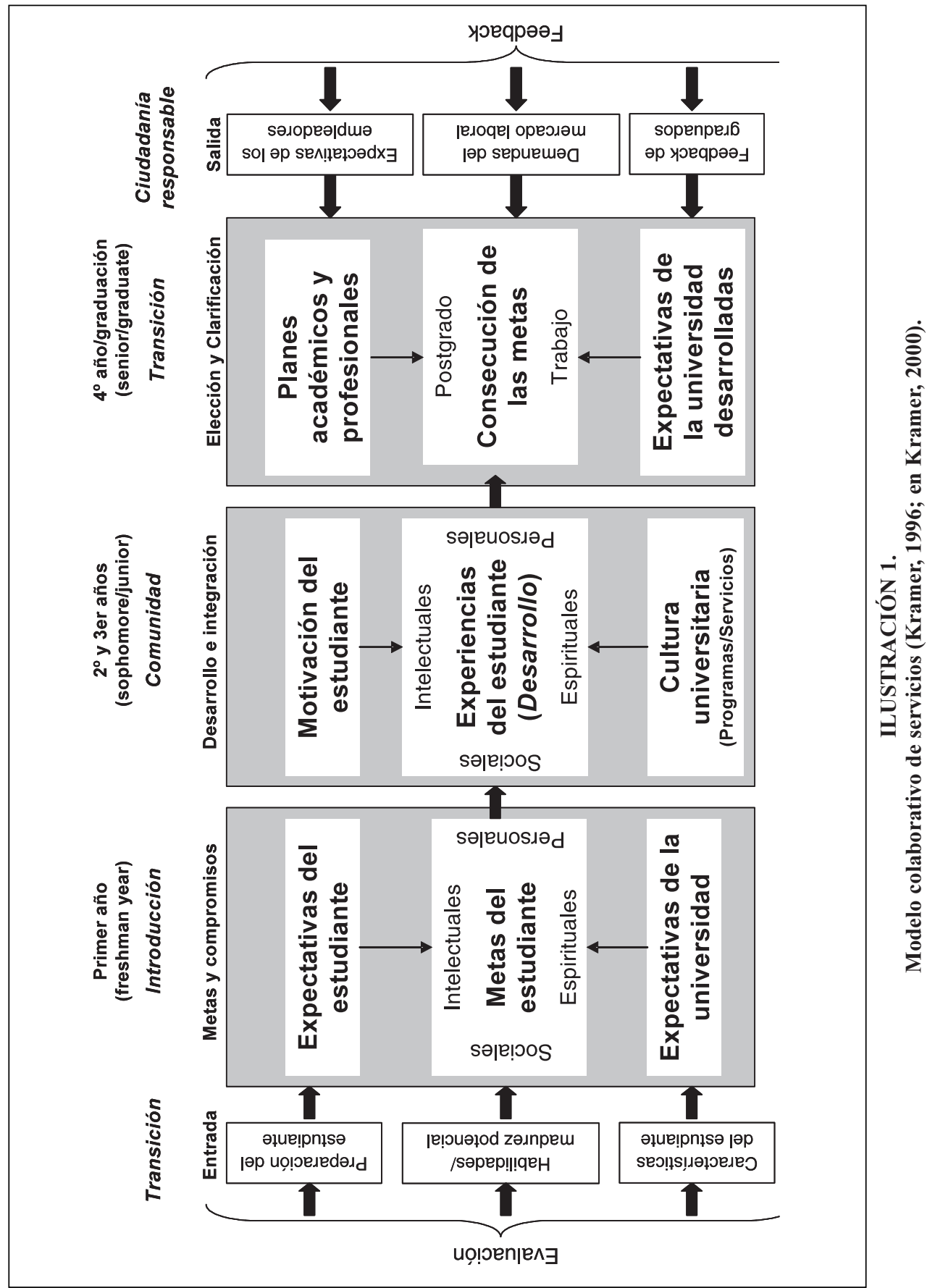


Según el modelo de Kramer, los orientadores académicos, profesionales especializados en diversos ámbitos de la orientación en las instituciones de educación superior de Estados Unidos, deben ser el eje central entre el estudiante y la variedad de servicios que ofrece el campus para ayudar a sus estudiantes (ayuda financiera, psicológica, orientación profesional, etc.). Es decir, los orientadores académicos deben ver la estructura del sistema de educación superior y la ayuda que este sistema ofrece desde la perspectiva del estudiante que transita a través de él (Kramer, 2000: 99). De acuerdo con este modelo, los estudiantes satisfechos con su experiencia educativa universitaria se caracterizan por haber tenido éxito académicamente y encontrarse comprometidos con un proyecto profesional futuro como resultado de haber recibido una adecuada información y orientación que comenzó en su primer año de estudios superiores.

El sistema de apoyo y orientación al estudiante en las instituciones de educación superior de Estados Unidos se caracteriza por la diversidad de opciones organizativas (Pardee, 2000). Esta diversidad es defendida por Sandeen (2001) al concluir que no existe un único modelo organizativo que sea válido en cualquier institución de educación superior. Entre los factores que afectan a la organización de este sistema destacan la misión o filosofía de la institución (según otorgue mayor prioridad a la investigación, al desarrollo profesional, al personal), las necesidades de los estudiantes (sociales, financieras, académicas), la actitud de los docentes hacia la orientación, el tamaño de la institución, el contexto y las relaciones de la institución con el exterior, y la relación del sistema de orientación y apoyo al estudiante con otras estructuras organizativas de la institución.

En el contexto europeo merece especial atención la creación en 1988 del Forum Européen de l'Orientation Académique (FEDORA), asociación establecida por profesionales implicados en la orientación, como impulsora de la orientación universitaria. Actualmente, FEDORA se encuentra muy implicada en el proceso de convergencia europea y en el papel de la orientación en este nuevo escenario. Bajo el auspicio de FEDORA, y financiado por la Comisión Europea como parte del programa Leonardo, Watts y Van Esbroeck realizan el que probablemente sea el estudio más citado sobre la organización y descripción de los servicios de orientación en las instituciones de educación superior europeas. En este estudio, denominado New skills for vocational guidance in higher education, Watts y Van Esbroeck (1998a) clasifican los servicios de orientación de las instituciones de educación superior europeas en función de las categorías propuestas en su modelo holístico de orientación centrado en las necesidades del estudiante (ver Ilustración 2).

En este modelo se diferencian tres ámbitos de actuación, académico, profesional y personal, que se estructuran a su vez en tres niveles. Mientras que el primer nivel está formado por el apoyo ofrecido a los estudiantes como parte de la función docente, el segundo y el tercer nivel se alejan progresivamente de los contextos de aprendizaje formal y adquieren mayor grado de especialización (Watts \& Van Esbroeck, 1998a; 1998b). En general, la ayuda ofrecida en el primer nivel suele ser más preventiva y encontrarse encaminada al desarrollo de habilidades que favorezcan la autonomía del estudiante, mientras que los niveles segundo y tercero tienden a organizarse a través de procesos especializados cada vez más desvinculados del proceso de enseñanza-aprendizaje y, por tanto, con un carácter más reactivo.

Según los autores, este modelo es compatible con diversas formas de organizar la orientación. No pretende imponer una estructura concreta sino maximizar las conexiones entre 




ILUSTRACIÓN 2.

Modelo holístico de orientación centrado en el estudiante (Watts y Van Esbroeck, 1998a, 1998b).

los distintos niveles y ámbitos, independientemente de la estructura que se adopte, con el fin de hacer un uso más eficiente de los recursos disponibles y mejorar el apoyo que recibe el estudiante.

En el ámbito universitario español, Álvarez Rojo (2000; citado en Rodríguez Moreno, 2002), propone un modelo similar al propuesto por Watts y Van Esbroeck, pero dando más importancia a la relación entre orientación y aprendizaje del alumnado universitario. El modelo integral de Álvarez Rojo se caracteriza por combinar la integración de los contenidos orientadores en las materias de estudio, con el sistema tutorial y los servicios de orientación universitaria con el objetivo fundamental de facilitar el desarrollo académico y profesional de los estudiantes y, si es posible y a la institución se le permite, fomentar el desarrollo personal y vital. Destacan como principales novedades en este modelo la integración de contenidos relacionados con la orientación en los planes de estudio y la priorización de los ámbitos de intervención en función de su necesidad, de forma que la institución debe dar apoyo académico-profesional pero se podría plantear hasta qué punto ha de intervenir en el ámbito personal. En cualquier caso, la institución debe comprobar mediante la evaluación si el sistema de orientación favorece el éxito académico de sus alumnos.

También en el ámbito español, la profesora Rodríguez Moreno (2002), tras analizar diversas propuestas de programas y modelos de intervención en orientación universitaria, entre ellos los de Watts y Van Esbroeck (1998a, 1998b) y Álvarez Rojo (2000), propone un modelo práctico e integral de orientación universitaria. Este modelo parte de ciertas premi- 
sas fundamentadas en la revisión de otros modelos: el modelo ha de ser evolutivo, exige la implicación del personal docente ya que, si es posible, es preferible que la orientación esté integrada en los programas curriculares, debería estar en conexión con los Consejos Sociales, requiere de profesionales especializados y considera la orientación individualizada como una actividad prioritaria en la trayectoria académica de un estudiante. El matiz más novedoso de esta aportación se encuentra en la autonomía de la universidad para diseñar su modelo por lo que no se proponen estructuras organizativas concretas, sino que se recomiendan una serie de intervenciones en función de dos dimensiones: el momento en el que se encuentre el estudiante y los clásicos ámbitos de intervención (académico, personal, fundamentalmente de autoconocimiento, y profesional) a los que se une específicamente la atención a la diversidad (alumnos discapacitados, adultos, exclusión social por género, etnia, entre otros).

Del análisis de estas propuestas organizativas provenientes de contextos diversos cabe destacar que aunque no es posible, ni deseable, definir una fórmula universal que nos diga cómo se debe organizar la orientación y el apoyo a los estudiantes en las instituciones de educación superior, es posible encontrar recomendaciones comunes en todas ellas, entre las que destacan:

- La orientación universitaria como sistema planificado. En los tres contextos (estadounidense, europeo y español) se considera necesario que la orientación se integre como un sistema planificado en la estructura organizativa de la institución.

- La institución factor clave en el modelo de orientación. Las características de los sistemas educativos y su tradición orientadora, el grado de institucionalización de sus servicios de orientación, la variedad en la tipología de sus estudiantes, son factores que definen a la institución y a su vez afectan a la organización del sistema de orientación.

- Orientación al estudiante ¿cuándo? y ¿en qué ámbitos? Las propuestas analizadas, algunas explícita y otras implícitamente, consideran que los estudiantes universitarios se enfrentan a problemas y situaciones muy diferentes dependiendo de en qué momento se encuentren: antes, durante, al finalizar o después de sus estudios. Por tanto, se enfatiza el carácter evolutivo en el proceso de orientación y la posibilidad de plasmarlo en la planificación concreta de un sistema de orientación. En cambio, la cuestión de los ámbitos de intervención no parece estar tan resuelta, sobre todo en el contexto español. No existe discusión sobre el deber de la universidad de ayudar en aspectos académicos y profesionales, pero ¿qué ocurre con el ámbito personal, psicológico y asistencial?, la respuesta de Álvarez Rojo en su propuesta es, sí se deben incluir pero si es posible y a la institución se le permite. Compartimos esta cautela basándonos en el principio previo del análisis de las necesidades de los estudiantes y de la institución como factor determinante del modelo de orientación.

- De lo preventivo a lo reactivo. Las propuestas analizadas ofrecen diversos niveles organizativos que empiezan en las propias aulas, con una intervención académica y de carácter preventivo, y terminan en servicios especializados con problemas de diversa índole. Estos niveles exigen una estructura coordinada de enlace entre las aulas, la facultad y los servicios especializados disponibles en el campus o en la comunidad.

- La orientación en el curriculum. Una de las funciones de la universidad es preparar a los estudiantes para acceder al mercado laboral o, simplemente, para ser más compe- 
tentes en el desarrollo de sus tareas cotidianas. La orientación en los planes de estudio supone formar a los estudiantes en competencias transversales (liderazgo, toma de decisiones, trabajo en equipo, etc.), es decir, útiles en cualquier contexto laboral y social. Un sistema de orientación planificado debe incorporar estos aspectos tan básicos para la auto-orientación académica, profesional y también personal en los programas académicos.

- La orientación, como sistema institucional planificado, debe ser evaluado. El sistema de orientación debe poder demostrar a la institución que contribuye a lograr sus fines.

\section{La Educación Superior}

\section{¿Cuáles son sus fines?}

Para conocer los fines de la educación superior conviene remontarnos al origen de las universidades europeas $\mathrm{y}$, en concreto, a la transformación que sufrieron en el siglo XIX con el nacimiento del Estado-nación que propició la diferenciación de tres modelos o arquetipos de universidad: el modelo alemán o humboltdiano, el francés o napoleónico y el británico o modelo Oxbridge (Mora, 2002a; Michavila y Calvo, 1998). Estos modelos de universidad persiguen objetivos diferentes. Mientras que en el modelo humboltdiano se persigue fomentar la investigación especializada y en el modelo napoleónico capacitar profesionalmente a los estudiantes, en el modelo británico se enfatiza la formación general, la educación liberal y el desarrollo personal de los estudiantes (Mora, 2002a; Rodríguez Espinar, 2004). Por otra parte, el modelo de universidad estadounidense ha recibido, en mayor o menor medida, influencias de los tres modelos occidentales europeos. El modelo británico ha dejado su huella en los colleges mientras que el modelo alemán ha influido notablemente en las universidades de investigación (Komives \& Woodard, 1996; Thelin, 2001).

Además de esta clasificación, se puede distinguir entre el modelo de universidad centrado en el contenido o modelo de producción industrial y el modelo centrado en el estudiante o de desarrollo de la capacidad de los individuos (Blimling, 2000; Mora, 2003). El primer modelo, el centrado en la producción industrial estaría más vinculado al modelo napoleónico mientras que el modelo de desarrollo de la capacidad y la libertad de los individuos se acercaría más al modelo Oxbridge.

Teniendo en cuenta las diferentes clasificaciones, no cabe duda de que nos encontramos en un momento de convergencia que favorece la consideración de un posible modelo único de universidad, universidad multifuncional según Porta (1998), universidad universal según Mora (2002a), que persigue fines comunes centrados en la persona aunque manteniendo las características y necesidades propias de un contexto determinado. Sin entrar en el debate de la posible consideración de un modelo único, lo cierto es que podemos hablar de objetivos universales que persigue la educación superior. En este sentido, diversos autores consideran que los principales fines de la universidad son la formación integral humana y la difusión de la cultura, la formación de profesionales acorde con las necesidades sociales y la dedicación a la enseñanza de la ciencia y la investigación científica (Comisión de las Comunidades Europeas, 2003 y 2005; Mora, 2003; Medina, 1996 y Bautista-Vallejo, 2000). Objetivos similares, aunque ampliados y más desarrollados, se recogen en el documento marco de la 
primera Conferencia Mundial de Educación Superior titulado Educación superior en el siglo XXI: visión y acción (UNESCO, 1998).

De las aportaciones señaladas podemos concluir que entre los objetivos que persigue la educación superior destacan enseñar, en el sentido amplio de favorecer el desarrollo personal dotando a las personas de las competencias necesarias para insertarse en el mercado laboral, desenvolverse en la sociedad y asumir una ciudadanía libre y responsable, investigar y promover el avance de la ciencia y el conocimiento, y servir tanto a la comunidad universitaria como a la sociedad en general potenciando valores democráticos, críticos y éticos. El sistema de orientación y apoyo al estudiante constituye una pieza institucional clave para la consecución de estos fines, especialmente, para favorecer el desarrollo de competencias que faciliten la inserción en el mercado laboral y para la formación de ciudadanos responsables.

\section{¿Hacia dónde vamos?}

En los últimos cuarenta años la educación superior, tanto en Europa como en otros lugares del mundo, ha sufrido una gran transformación. Desde el marco social, fenómenos como la globalización, los cambios derivados de la sociedad de la información, la revolución tecnológica de las comunicaciones, la configuración del trabajo basado en la gestión del conocimiento, el nuevo marco organizativo del trabajo dominado por la caducidad rápida de los productos y las ideas, entre otras causas, producen nuevas necesidades de formación que afectan al sistema de educación superior (Rodríguez Espinar, 2004; Teichler, 1999; Watts \& Van Esbroeck, 2000; Taylor, 2003 y Sporn, 2003).

Diversos estudios han revisado las tendencias futuras de la educación superior y los retos a los que se enfrenta (Eurydice 2000 y Teichler, 1999). Cabe destacar en la recopilación de Teichler (1999) la importancia otorgada al sistema de orientación al referirse a los retos a los que se enfrenta la educación superior. Teichler apuesta por el aprendizaje y la socialización, más allá de la instrucción que se recibe en el aula, mediante la experiencia práctica en el mercado laboral nacional e internacional, la comunicación fuera del aula entre estudiantes y profesores y la utilización de servicios de orientación, entre otros.

A continuación, detallamos el estado actual de algunos de los cambios más significativos que se han producido, fundamentalmente, en la educación superior europea.

\section{Sociedad del conocimiento y diversidad en la población de estudiantes}

El cambio de una sociedad basada en la producción a otra fundamentada en el conocimiento ha animado a un mayor número de personas a realizar estudios superiores. Lo que algún día fue un sistema universitario orientado a un grupo élite que cubría el 5\% de las cohortes de jóvenes se ha transformado en un sistema de masas que cubre entre un $20 \%$ y un $30 \%$ de esas cohortes, además de incluir a muchos más estudiantes adultos (Watts \& Van Esbroeck, 2000). Este incremento en el número de estudiantes ha favorecido la variedad de su perfil. Ha aumentado la participación de alumnos con discapacidad y con necesidades educativas especiales, alumnos de minorías étnicas y de clases desfavorecidas, alumnos que trabajan a tiempo parcial, estudiantes internacionales, mayores de veinticinco años, estudiantes adultos motivados por una mejor formación para su trabajo y estudiantes a distancia 
gracias al desarrollo de las tecnologías de la información y de la comunicación (Watts \& Van Esbroeck, 1998b; Eurydice, 2000 y 2002).

Otro factor de gran preocupación en la educación superior que genera diferencias entre los estudiantes es el bajo rendimiento académico, especialmente en los primeros cursos y en las enseñanzas técnicas. Este problema, aunque es menos acusado en algunos países como Alemania, Grecia o Noruega, afecta a todos los países de la OECD que presenta una tasa media de abandono del 20\%. En España este porcentaje asciende notablemente hasta situarse en torno al 30\% (OECD, 2005).

\section{Un mercado laboral cambiante}

La transformación de sociedades basadas en la producción a sociedades fundamentadas en la información y el conocimiento, y la consideración de un mercado europeo único, entre otros factores, han favorecido la consolidación de un mercado laboral flexible y en constante cambio. En un mercado laboral de estas características se demandan competencias genéricas más que conocimientos específicos (Mora, 2002b; Teichler, 1999). Según la opinión de graduados y empleadores, entre las competencias más necesarias en el mercado laboral se encuentran las habilidades interpersonales, junto con habilidades básicas como puede ser el manejo del ordenador, quedando en un segundo plano los conocimientos generales. Por el contrario, la percepción de los graduados y de los empleadores contrasta con la de los académicos quienes opinan que los jóvenes graduados necesitan fundamentalmente la adquisición de conocimientos generales mientras que las habilidades interpersonales pasarían a los últimos lugares de su ranking de competencias demandadas (Mora, 2002b, Rodríguez Moreno, 2002). Para que la universidad se adecue a las demandas del mercado de trabajo será necesario que la formación tienda a ser cada vez más general y práctica sustituyendo la actual formación excesivamente teórica y especializada. La reforma curricular prevista en el diseño de los planes de estudio en el marco de la convergencia europea insiste en este cambio en el proceso de enseñanza-aprendizaje centrando el interés en el alumno y en su proceso activo de aprendizaje.

\section{La rendición de cuentas}

La expansión del número de estudiantes no ha ido acompañada de ingresos proporcionados del gasto público en educación superior. En los sistemas de educación superior europeos, predominantemente públicos, se otorgó a partir de las décadas de los setenta y los ochenta mayor autonomía a las instituciones de manera que el Estado, la principal fuente de financiación de las universidades, ha pasado de controlar el proceso a controlar la calidad del producto (Neave \& Van Vught, 1994). En consecuencia, se ha exigido a las instituciones que rindan cuentas del desempeño de sus funciones de acuerdo con objetivos y criterios establecidos a través de diversos procedimientos de evaluación: acreditación de programas, evaluación institucional, certificación, etc. (Hämäläinen, Pehu-Voima, \& Wahlén, 2001; Mora, 2002a; Mora y Vieira, 2005). La rendición de cuentas por parte de las instituciones de educación superior ha sido mucho más tardía en Europa que en Estados Unidos, al estar mayoritariamente controlada por el Estado (Sebkova, 2002). 


\section{El Espacio Europeo de Educación Superior}

El Espacio Europeo de Educación Superior comenzó a forjarse con la Magna Charta Universitatum de 1988 firmada en Bolonia por rectores de universidades europeas en la que se enfatizaba la importancia de la autonomía universitaria como condición para adaptarse a los requerimientos cambiantes de la sociedad (EUA, 2001). La puesta en marcha en 1989 de los programas de movilidad interuniversitaria de estudiantes Erasmus (1989-1994) y Sócrates/Erasmus (1995-2006), favorecieron la creación de este espacio común.

Posteriormente, la consolidación definitiva del Espacio Europeo de Educación Superior ha sido consecuencia de las declaraciones de los ministros europeos de educación en La Sorbona (1998), Bolonia (1999), Praga (2001), Berlín (2003) y Bergen (2005). En concreto, la Declaración de Bolonia instaba a los sistemas europeos de educación superior a ser más comparables, compatibles, transparentes y flexibles (Mora, 2002a). Como consecuencia de estas declaraciones han tenido lugar dos hechos trascendentales: (a) la transformación de los programas de estudio en casi todos los países europeos para adaptarlos antes del 2010 a una estructura Bachelor/Master que debe ser común para todos los países europeos y (b) la creación en la mayoría de los países europeos de organismos, agencias, consejos, cuyos principales objetivos han sido evaluar la calidad de la educación superior y establecer un sistema común europeo de garantía de calidad (Haug, 1999).

Tanto la movilidad de estudiantes como la transformación de los programas de estudio han favorecido el desarrollo de los sistemas de orientación. Según el Informe Trends III (Reichert y Tauch, 2003), la creciente movilidad interuniversitaria de estudiantes en el Espacio Europeo de Educación Superior ha favorecido la mejora de las condiciones de movilidad para los estudiantes, especialmente en la oferta de servicios de orientación e información para alumnos internacionales. Los resultados de este informe indican que los servicios de recepción y de orientación han mejorado en más del 75\% de las instituciones europeas de educación superior, junto con otros servicios de asesoramiento, actividades sociales y culturales para estudiantes extranjeros, alojamiento, tutorías académicas e información sobre la oferta académica en otras instituciones. Posteriormente, el Informe Trends $I V$ (Reichert y Tauch, 2005) pone de manifiesto la necesidad de que las universidades prevean que debido a la reforma curricular centrada en el estudiante en la que se enfatiza un proceso de enseñanza-aprendizaje más flexible, los estudiantes necesitarán más información y una orientación más adecuada para tomar decisiones y elegir su proyecto académico y profesional.

Por otra parte, en el contexto de la convergencia europea se han realizado proyectos de innovación educativa, que han demostrado los efectos positivos del apoyo al estudiante en los procesos de enseñanza-aprendizaje en el rendimiento del estudiante. En estos proyectos, se propone una metodología encaminada a potenciar el aprendizaje del alumnado, fomentar competencias genéricas mediante la coordinación del equipo de profesores, la atención individualizada al estudiante, y la planificación de la tutoría, entre otros aspectos.

\section{El estudiante como cliente}

Los cambios apuntados en la educación superior han favorecido la consideración del alumno como cliente de la institución (Quintanilla, 1998; Coaldrake, 2001; Melear, 2003; 
Mosier \& Schwarzmueller, 2002). En España, al igual que en otros países, la consideración del estudiante como cliente que debe estar satisfecho con la institución ha sido propiciada por el temor de muchas universidades, sobre todo con algunas titulaciones poco demandadas, al ver descender el número de alumnos matriculados año tras año. Además de esta reducción en la demanda de determinadas titulaciones, los estudiantes de educación superior son cada vez más exigentes con la calidad percibida por su dinero, en consecuencia, esperan encontrar en el campus un conjunto de servicios dedicados a atender sus necesidades. La importancia otorgada por gestores e investigadores a la opinión del estudiante como cliente de la institución queda patente en el interés relativamente reciente por encuestar a los estudiantes con el fin de conocer cómo ha sido su experiencia en las instituciones de educación superior (Green, Brannigan, Mazelan, \& Giles, 1994). Según el estudio realizado por Hill, Lomas y MacGregor (2003), los factores más valorados por los estudiantes para recibir una educación de calidad fueron el profesor y los sistemas de apoyo al estudiante.

En síntesis, podemos concluir que la educación superior ha experimentado enormes cambios en las últimas décadas. Nuestra pretensión en este apartado ha sido conocer las tendencias actuales de la educación superior con el fin de analizar hasta qué punto las instituciones de educación superior precisan de mecanismos de apoyo a sus estudiantes para adecuarse a estas tendencias.

\section{Educación superior: ventajas e inconvenientes para el desarrollo de la orientación universitaria}

En este apartado, abordamos la necesidad de planificar y establecer sistemas de orientación en las universidades partiendo de la situación actual de la educación superior. Por una parte, se da respuesta a los problemas que afectan al desarrollo de la orientación universitaria y, por otra, se analiza su importancia de acuerdo con las tendencias señaladas para la educación superior.

\section{Las principales funciones de la universidad son instruir e investigar}

El modelo de universidad centrado en el contenido todavía perdura en algunos sectores desde los que se defiende que el único deber de la universidad, cuna del conocimiento, consiste en instruir, en el sentido de aumentar el conocimiento de los estudiantes, e investigar o promover el cambio cualitativo y cuantitativo de ese conocimiento (Blimling, 2000). En un sistema de estas características, la orientación, entendida como sistema de apoyo a lo largo de la vida y parte fundamental del proceso de enseñanza-aprendizaje, tiene poca cabida. En general, el profesorado que asume esta idea de universidad no comprende que una de sus funciones, además de investigar y enseñar un contenido determinado, es tutorizar y ayudar a sus alumnos. La instauración de un sistema de orientación en las universidades se encuentra con un problema de cultura docente que excluye la orientación como parte integral del proceso de enseñanza-aprendizaje y como un derecho del estudiante.

Los fines que persigue la educación superior en la actualidad se aproximan más al modelo centrado en el estudiante que al centrado en la producción industrial (Blimling, 2000; Mora, 2003), principalmente porque en una sociedad fundamentada en el conocimiento y 
caracterizada por un mercado laboral flexible y cambiante es fundamental el desarrollo de diversas capacidades y competencias que permitan a las personas adaptarse a los cambios que ésta demanda. El sistema de orientación y apoyo al estudiante universitario se encuentra comprometido con el modelo de educación superior fundamentado en el desarrollo de la capacidad del estudiante, en el desarrollo de su responsabilidad, sus competencias interpersonales, el trabajo en equipo, el respeto y la confianza (Blimling, 2000; ACPA, 1996).

En este sentido, la ACPA (American College Personnel Association) publicó en 1996 el documento The Student Learning Imperative: Implications for Student Affairs con la intención de estimular un debate sobre cómo los profesionales responsables del sistema de apoyo al estudiante pueden crear las condiciones adecuadas para fomentar la mejora del aprendizaje y del desarrollo personal del estudiante. Estas condiciones se fundamentan principalmente en dos asunciones básicas:

1. Los conceptos aprendizaje, desarrollo personal y desarrollo del estudiante son inseparables. La educación superior tradicionalmente ha organizado sus actividades separando academic affairs o asuntos académicos (aprendizaje, curriculum, desarrollo cognitivo) y student affairs (actividades extracurriculares, asociaciones de estudiantes, desarrollo afectivo y personal). Sin embargo, esta dicotomía resulta irrelevante al finalizar los estudios superiores ya que el desempeño en un puesto de trabajo, en la vida familiar, en las actividades comunitarias, depende de competencias tanto cognitivas como afectivas.

2. El entorno puede ser diseñado intencionadamente para promover el aprendizaje del estudiante. Por ejemplo, los estudiantes aprenden más cuando sus profesores organizan el aula para promover la colaboración y la participación. De forma similar, cuando los profesionales vinculados al sistema de orientación animan a los estudiantes a utilizar recursos institucionales (bibliotecas, organizaciones de estudiantes, laboratorios, etc.) y a participar en la gestión de la universidad y en otras actividades educativas, los estudiantes adquieren ciertas competencias genéricas muy necesarias en el mercado laboral actual.

Estas condiciones necesarias para el adecuado funcionamiento del sistema de orientación en las universidades se encuentra, entre otros, con un problema básico: la resistencia del profesorado a dedicar más tiempo del estrictamente necesario a su alumnado cuando la dedicación a la investigación le reporta mayores beneficios.

\section{La autonomía del alumnado}

Una de las razones más utilizadas para argumentar la poca importancia de la orientación en las instituciones de educación superior reside en la autonomía y madurez que se supone tiene el estudiante universitario en la toma de decisiones (Sanz García, 2001; Watts \& Van Esbroeck, 2000). Se considera que en esta etapa los alumnos son personas adultas, independientes, que han decidido libremente proseguir con estudios superiores, y por tanto, deben asumir y ser capaces de afrontar por sí mismos y sin ayuda adicional sus estudios y su vida en general.

En realidad, el paso por la universidad puede ser considerado como una de las transiciones con mayores consecuencias para el desarrollo profesional, social, personal y emocional 
de los alumnos. Éstos necesitan que la universidad les preste su ayuda para poder afrontar esta transición y les capacite para futuras transiciones a las que deberán hacer frente a lo largo de su vida (Rodríguez Moreno, 2002). Además, la autonomía del alumnado varía, por una parte, a medida que aumenta la diversidad de éste y sus características (etnia, lengua, religión, cultura, capacidad, etc.) y, por otra, en la medida en que se producen cambios sustanciales en los sistemas de educación superior, situación que se aproxima actualmente en Europa.

Tanto la variedad del alumnado como los cambios de los sistemas de educación superior, implican la necesidad de orientación e información actualizada en un formato adaptado a las necesidades concretas de los diferentes colectivos de estudiantes. Esto no quiere decir que el hecho de que los alumnos puedan contar con tutores y con un conjunto de servicios diseñados para ayudarles signifique que se está impidiendo la capacidad del alumnado de desarrollar su autonomía. Al contrario, y como afirma Ponz (1996: 88), la Universidad en que se valora a la persona no puede desentenderse de hacer cuanto esté en su mano por favorecer el desarrollo de la personalidad de cuantos integran la comunidad universitaria, se sabe responsable de la educación de las personas aunque sus estudiantes tengan ya 18 o más años, y afronta esa responsabilidad de la forma apropiada a esa edad y, añadimos, a las características personales de cada alumno.

\section{Demostrar los efectos positivos de la orientación}

La orientación no ha sido considerada elemento imprescindible en las instituciones de educación superior porque no ha demostrado sus efectos positivos. Álvarez Rojo (2000, en Rodríguez Moreno, 2002: 64) indica que la presencia de servicios de orientación en las universidades es un indicador de calidad, siempre y cuando una universidad demostrara que consigue que sus alumnos alcancen niveles óptimos de rendimiento en el aprendizaje a través de la buena organización de los servicios de orientación académica y la tutoría. Existe un gran volumen de investigación evaluativa sobre los resultados académicos de los estudiantes, pero pocas de estas investigaciones demuestran la influencia del sistema de orientación en estos resultados (Blimling, 2000).

Diversas investigaciones realizadas en Estados Unidos ponen de manifiesto la importancia de todas las funciones relacionadas con el sistema de orientación y apoyo al estudiante, tanto de las funciones que hemos denominado clave como de las de soporte. En concreto, las investigaciones de Astin (1993), Pascarella y Terenzini (1991) y Pace (1980), entre otros, indican que ciertas experiencias desarrolladas por los estudiantes fuera del aula influyen en el rendimiento académico y la persistencia (Terenzini, Pascarella \& Blimling, 1999). El término experiencias fuera del aula (out-of-class experiences) hace referencia a las actividades que se realizan en las instituciones de educación superior no incluidas en las clases o actividades instruccionales programadas para un curso académico de una titulación determinada. Entre las experiencias fuera del aula que tienen un efecto positivo en el rendimiento $\mathrm{y}$ la persistencia destacan:

a) recibir asesoramiento académico,

b) trabajar a tiempo parcial en el campus, 
c) interactuar con otros estudiantes y con profesores, principalmente cuando se tratan temas académicos,

d) socializarse con personas de otras razas o grupos culturales,

e) tener un trabajo en prácticas,

f) estudiar un trimestre en el extranjero,

g) realizar trabajos comunitarios y

h) vivir en una residencia universitaria, en especial en aquellas cuya política incluye la atención al estudiante (Kuh, Gonyea, \& Palmer, 2001).

Por supuesto, además de las actividades realizadas fuera del aula, las actividades realizadas en el aula y las características del profesor influyen a su vez en el rendimiento y la persistencia. Así, Chickering \& Gamson (1987) consideran determinantes del rendimiento académico el contacto con los profesores, la cooperación entre los estudiantes, el aprendizaje activo, el feedback inmediato, las altas expectativas y el respeto por diversas formas de aprendizaje. Asimismo, incluyen entre los factores que favorecen el rendimiento académico en la universidad las actividades que promueven que los estudiantes se sientan incluidos, pertenecientes a un grupo y donde las expectativas de lo que hay que hacer están claras y suponen un reto razonablemente alto. En esta línea el programa SEEK (Search for Education, Elevation and Knowledge) se apoya en la hipótesis de que el rendimiento académico universitario se ve afectado por una serie de variables endógenas (utilización de tutorías y servicios de remediación, desarrollo de estrategias básicas, uso de servicios de orientación, participación en asociaciones de estudiantes e integración en la cultura universitaria), variables exógenas (rendimiento académico previo, acceso a la universidad, características familiares y personales) y variables de interacción con la institución (Francis, Nelly y Bell, 1993; citado en Pozo Muñoz, 2000).

La importancia de estos resultados para justificar la necesidad de sistemas de orientación y apoyo al estudiante radica en que las funciones de este sistema están vinculadas con actividades, tanto fuera como dentro del aula, que inciden positivamente en el rendimiento y en el desarrollo personal. En este sentido, las funciones ofrecidas por el sistema de orientación son evaluadas y se utilizan como indicadores de calidad de las instituciones de educación superior. En resumen, las funciones del sistema de orientación favorecen el éxito académico, como apoyo que deben ofrecer los profesores, los profesores-tutores y otros profesionales vinculados al asesoramiento académico, y también, en la creación de espacios y experiencias que inviten al alumno a expresar sus opiniones, contar sus problemas, realizar actividades extra-curriculares y en definitiva, sentirse parte integrante de una organización.

\section{Aunque la orientación tenga efectos positivos ¿es un extra?}

Las instituciones se han encontrado con recursos económicos limitados y recortes en sus presupuestos. Ante esta situación, la pregunta que se plantean las instituciones es ¿son esenciales los servicios de orientación para el funcionamiento efectivo de la institución? Así, ante el planteamiento de dónde recortar gastos, el sistema de orientación y apoyo al estudiante ha sido uno de los más afectados (Watts \& Van Esbroeck, 2000). Tanto la necesidad de demostrar los efectos positivos del sistema de orientación, como la cuestión de si este sistema resulta esencial o puede ser considerado un extra a la hora de distribuir fondos, ha 
desencadenado la necesidad del sistema de orientación y apoyo al estudiante de rendir cuentas y demostrar que su labor resulta efectiva para conseguir los fines que se propone la institución y que, por ello, debe contar con los recursos necesarios (Blimling, 2000; Watts \& Van Esbroeck, 2000).

Aunque diversas investigaciones han demostrado el efecto positivo de ciertas funciones vinculadas al sistema de orientación, no cabe duda de que la multitud de funciones que constituyen este sistema no pueden ser igual de importantes. Si una institución se planteara qué servicios de orientación se puede permitir y de cuáles podría prescindir, es posible que realizara una clasificación similar a la que hemos ofrecido en base a los propios fines que persigue la institución. Es decir, todas aquellas funciones que fomentan el aprendizaje y el desarrollo del estudiante se deben mantener, mientras que otras funciones de soporte de índole asistencial pueden ser cuestionables.

Aunque esto sea cierto, no debemos olvidar un aspecto señalado al hablar de las tendencias actuales de la educación superior. La consideración del estudiante como cliente al que se debe satisfacer está muy relacionada con la oferta de funciones de soporte que atiendan a las distintas necesidades y expectativas de los estudiantes. En este sentido, la universidad debe evaluar las necesidades que detecta entre sus clientes, los alumnos, para tratar de responder adecuadamente a ellas (Sanz García, 2001: 109). En esta línea la Comisión de las Comunidades Europeas (2005) insiste en convertir a las universidades europeas en una referencia a nivel internacional introduciendo mejoras con el fin de aumentar el atractivo de las mismas para competir con las de otros países desarrollados, concretamente con las de Estados Unidos. Este objetivo será muy difícil de lograr si Europa no hace un gran esfuerzo por organizar sus instituciones en torno al estudiante y a sus necesidades.

\section{En España, predominancia del modelo de servicios}

En España, la falta de legislación en el ámbito universitario en materia de orientación y la proliferación rápida de servicios de atención al estudiante, ha ocasionado que la organización de la orientación en las universidades se aleje del concepto de sistema institucionalizado.

No obstante, la ausencia de un marco legislativo no ha sido óbice para iniciar el proceso de institucionalización de la orientación universitaria que comenzó a finales de los sesenta con actividades orientadoras de corte psicotécnico en la Universidad Autónoma de Madrid y continuó poco después en la Universidad de Barcelona. Estas experiencias pioneras y la preocupación de un grupo de profesionales y académicos por analizar la necesidad de orientación de los estudiantes universitarios (entre ellos destacan Díaz Allué, M. T., 1973 y 1989; González Simancas, J. L., 1973, Sanz Oro, R., 1982, Rodríguez Moreno, M. L., 1990, en Echeverría, 1997) supusieron los primeros pasos de un proceso de sensibilización institucional por la orientación universitaria. En al ámbito de la orientación profesional, se crearon a partir del curso 1975/76 los Centros de Orientación e Información de Empleo (COIE) en las universidades como servicios externos a los centros con el fin de atender las necesidades de orientación laboral del alumnado (Alonso Rivas y López Quero, 1983).

En la década de los noventa, la puesta en marcha en España de planes institucionales de evaluación de la calidad de las universidades promovió el inicio de acciones de mejora rela- 
cionadas con la acción tutorial en el ámbito institucional. De hecho, muchas de las acciones de mejora que fueron premiadas en las convocatorias de 2000, 2001 y 2002 del Plan Nacional de Evaluación de la Calidad de las Universidades I y del Plan de Calidad de las Universidades II se refieren a la puesta en marcha de planes de acción tutorial que se encuentran en la actualidad en una fase exploratoria (Michavila y García Delgado, 2003).

Entre los problemas que se han encontrado las universidades para el buen funcionamiento de los planes de acción tutorial, los más relevantes hacen referencia a la organización de los mismos en base a la colaboración voluntaria del profesorado, lo que genera problemas en un sistema que prima la investigación y no la docencia, y la necesidad de formación previa y específica del profesorado. Entre las sugerencias aportadas para mejorar su funcionamiento destaca la necesidad de incluir los planes de acción tutorial en los planes estratégicos de las universidades y en contratos-programa, la búsqueda de estrategias para que los estudiantes valoren y utilicen convenientemente los dispositivos de orientación y tutoría, la introducción de las tecnologías de la información y la comunicación y la consideración de la tutoría como indicador de calidad y posible requisito de acreditación (Michavila y García Delgado, 2003).

Por tanto, el modelo de orientación predominante en las universidades españolas se caracteriza, por una parte, por la consolidación de servicios especializados, fundamentalmente de orientación profesional y de información académica, independientes de las facultades o centros universitarios $\mathrm{y}$, por otra, por la puesta en marcha de planes de acción tutorial vinculados a los centros pero poco o nada relacionados con los servicios universitarios de orientación (Echeverría, 1996 y 1997; Sánchez García, 1998; Vidal, Díez y Vieira, 2003). No obstante, se ha dado un gran paso desde que comenzaron los procesos de evaluación en las universidades, ante los cuales se encontraron muchas resistencias, hasta el momento actual en el que los centros universitarios toman la iniciativa de poner en marcha actuaciones para mejorar la situación de los alumnos, como ha sido el caso de los planes de acción tutorial. Esta preocupación por el bienestar y el rendimiento académico del estudiante se produce en un momento en el que las universidades españolas se sienten amenazadas por el descenso del número de estudiantes y por la creciente competencia entre universidades por la captación de alumnos, lo que lleva a tratar de ofrecerles más servicios. En este contexto, la tutoría y los servicios a los estudiantes son percibidos como factor estratégico para la mejora de la calidad del principal servicio que presta la universidad: el formativo (Michavila y García Delgado, 2003; Rodríguez Espinar, 2004).

En resumen, el aspecto más positivo de la situación actual del sistema de orientación a los estudiantes en las universidades españolas es la reflexión que se está realizando sobre su organización en un marco planificado e institucionalizado en un momento de profundos cambios en el sistema de educación superior acordes a la consolidación del Espacio Europeo de Educación Superior.

\section{Conclusiones}

En la educación superior, la orientación a los estudiantes debe ser entendida como aquellas funciones planificadas en un sistema institucional de orientación y apoyo al estudiante. En el análisis de diversas propuestas organizativas de este sistema destaca la coincidencia 
de ciertos elementos organizativos básicos: el análisis previo de las necesidades de la institución, el carácter evolutivo de las funciones de orientación (antes, durante y después de los estudios) y la atención a diversos ámbitos (académico, profesional y personal-asistencial). Además, la mayoría de las propuestas coinciden en señalar el carácter eminentemente preventivo al que atiende la orientación y, por tanto, la importancia de que estas funciones formen parte del propio currículum académico.

En la actualidad, el contexto en el que se establece el sistema de orientación se halla inmerso en un momento de profundos cambios liderados, en nuestro entorno, por el proceso de construcción del Espacio Europeo de Educación Superior. En este proceso confluyen multitud de factores: globalización, competitividad, comparabilidad, movilidad, exigencias de garantía de calidad, adaptación a la sociedad del conocimiento y a la diversidad de los estudiantes, modificación de planes de estudio y de metodologías, y con todo ello, un importante giro en la planificación del sistema universitario hacia las características de los estudiantes. Nos encontramos, por tanto, ante un modelo de universidad centrado en el estudiante, teniendo en cuenta las diferencias existentes entre sistemas educativos que responden a los clásicos arquetipos, en el que las universidades europeas están diseñando una metodología docente capaz de ofrecer una formación integral acorde a las necesidades de una sociedad sin fronteras. Para responder a este modelo, el sistema de orientación y apoyo al estudiante constituye una pieza institucional clave, que debe ser objeto de debate en el diseño del Espacio Europeo de Educación Superior, en cuya planificación y organización son fundamentales cuatro recomendaciones:

1) la visión institucional centrada en el estudiante debe reflejarse en los planes estratégicos de las instituciones,

2) coordinación de las funciones destinadas a informar, orientar y atender a las necesidades de los estudiantes en un único sistema de orientación y apoyo al estudiante institucional,

3) el sistema de orientación y apoyo de las instituciones de educación superior debe responder a las características y necesidades de los estudiantes y

4) la evaluación institucional debe incluir al sistema de orientación y apoyo al estudiante.

En el contexto universitario español, la situación de los mecanismos de orientación y atención al estudiante se caracterizan por su escasa planificación y coordinación en torno a fines comunes. Teniendo en cuenta esta situación de la orientación en la universidad española, y aprovechando que ésta se encuentra implicada en la consolidación del Espacio Europeo de Educación Superior estructurado en torno a un concepto de crédito que prima el aprendizaje del estudiante, nos encontramos en un momento idóneo, y quizá único, para que los responsables de las instituciones de educación superior reflexionen y planifiquen un sistema de orientación y apoyo al estudiante a la hora de definir tanto las reformas de los planes de estudio como la planificación estratégica de las universidades.

\section{Referencias bibliográficas}

ACPA. (1996). The student learning imperative: Implications for student affairs. Washington, DC: American College Personnel Association. 
Alonso Rivas, J., y López Quero, M. (1983). Los COIE de las universidades madrileñas: seis años de actividades. Madrid: Fundación Universidad-Empresa.

Alonso Tapia, J. (1995). Orientación educativa. Teoría, evaluación e intervención. Madrid: Síntesis.

Álvarez González, M. y Bisquerra Alzina, R. (Coords.) (1996). Manual de orientación y tutoría. Barcelona: Praxis.

Álvarez Rojo, V. (1994). Orientación educativa y acción orientadora: relaciones entre la teoría y la práctica. Madrid: EOS.

Bautista-Vallejo, J. M. (2000). La universidad en la encrucijada de la formación. La tensión permanente por la mejora de la calidad. Huelva: Herqué Editores.

Bisquerra, R. (1998). Modelos de orientación e intervención psicopedagógica. Barcelona: Praxis.

Blimling, G. S. (2000). Accountability for Student Affairs: Trends for the $21^{\text {st }}$ Century. En C. Johnson, $\&$ H. Cheatham (Eds.), Higher Education trends for the next century: a research agenda for student success. American College Personnel Association. (Disponible en: www.acpa.nche.edu/seniorscholars/trends/trends.htm; Consultado 15-12-04).

Caselles, J. M. (2003). Gestión por procesos: innovación y mejora. Ponencia presentada en el XXVII Congreso Nacional de Estadística e Investigación Operativa. Lleida, abril 2003.

Chickering, A. W., \& Gamson, Z. F. (1987). Seven Principles for Good Practice in Undergraduate Education. AAHE Bulletin, 39(7), 3-7.

Coaldrake, P. (2001). Responding to changing student expectations. Higher Education Management, 13 (2), 75-92.

Comisión de las Comunidades Europeas. (2003). El papel de las universidades en la Europa del conocimiento. Bruselas: CCE.

Comisión de las Comunidades Europeas. (2005). Movilizar el capital intelectual de Europa: crear las condiciones necesarias para que las universidades puedan contribuir plenamente a la estrategia de Lisboa. Bruselas: CCE.

Díaz Allué, M. T. (1989). La Orientación universitaria ante la problemática académica y profesional del estudiante. Madrid: Narcea.

Echeverría, B. (1996). Jornadas sobre orientación académica y profesional en la Universidad. Barcelona: Universitat de Barcelona.

Echeverría, B. (1997). Los servicios universitarios de orientación. En P. Apodaca y C. Lobato (Coords.), Calidad en la Universidad: Orientación y Evaluación. (pp. 112-136). Barcelona: Laertes.

EUA. (2001). Quality Assurance in Higher Education. A policy paper of the European University Association. Brussels: European Universities Association.

Eurydice. (2000). Two decades of reform of Higher Education in Europe: 1980 onwards. Brussels: EURYDICE European Unit.

Eurydice. (2002). Educación Superior. En Eurydice, Las cifras clave de la educación en Europa. Brussels: Eurydice.

Green, D., Brannigan, C., Mazelan, P., \& Giles, L. (1994). Measuring student satisfaction: a method of improving the quality of the student's experience. In S. Hazelgrove (Ed.), The Student Experience. Buckingham: Open University Press.

Hämäläinen, K., Pehu-Voima, S., \& Wahlén, S. (2001). Institutional Evaluations in Europe. Helsinki: ENQA.

Haug, G. (1999). Trends and Issues in Learning Structures in Higher Education in Europe. Confederation of European Union Rectors' Conferences and the Association of European Universities. 
Hill, Y., Lomas, L., \& MacGregor, J. (2003). Students' perceptions of quality in higher education. Quality Assurance in Education, 11(1), 15-20.

Jiménez Gámez, R. y Porras Vallejo, R. (1997). Modelos de acción psicopedagógica: entre el deseo y la realidad. Málaga: Algibe.

Kells, H. R. (1997). Procesos de autoevaluación: una guía para la autoevaluación en la educación superior. Perú: Fondo Editorial de la PUCP.

Komives, S. R., \& Woodard, D. B. (1996). Building on the past, shaping the future. En S. R. Komives, \& D. B. Woodard, Student Services: A Handbook for the Profession (pp. 536-555). San Francisco, CA: Jossey-Bass.

Kramer, G. L. (2000). Advising students at different educational levels. En V. N. Gordon, \& W. R. Habley (Eds.), Academic Advising. A comprehensive handbook (pp. 84-104). San Francisco: JosseyBass.

Kuh, G. D., Gonyea, R. M., \& Palmer, M. (2001). The disengaged commuter student: fact of fiction? Commuter Perspectives, 27(1), 2-5.

Kuh, G., \& Schuh, J. (1991). Some good news about campus life. Change, 23(5), 48-56.

Ludeman, R. B. (2001). The role of student affairs and services in Higher Education: A practical manual for developing, implementing, and assessing student affairs programs and services. IASAS/UNESCO.

Martínez Clares, P. (2002). La orientación psicopedagógica: modelos y estrategias de intervención. Madrid: EOS.

Medina, R. (1996). Los fines de la universidad. En V. García Hoz (Dir.), La educación personalizada en la universidad (pp. 131-154). Madrid: Ediciones Rialp.

Melear, K. B. (2003). From in loco parentis to consumerism: a legal analysis of the contractual relationship between institution and student. The NASPA Journal, 40 (4), 124-148.

Michavila, F. y Calvo, B. (1998). La universidad española hoy. Propuestas para una política universitaria. Madrid: Editorial Síntesis.

Michavila, R. y García Delgado, J. (2003). La tutoría y los nuevos modos de aprendizaje en la universidad. Comunidad de Madrid: Cátedra UNESCO.

Mora, J.-G. (2002a). Evaluación y acreditación de los estudios universitarios españoles en el contexto europeo. En F. Michavila y S. Zamorano (Eds.), Acreditación de las enseñanzas universitarias: un futuro de cambio (pp. 39-54). Comunidad de Madrid: Consejería de Educación. Dirección General de Universidades.

Mora, J.-G. (2002b). La conexión entre la Universidad y la Empresa. El empleo universitario y los perfiles profesionales. Conferencia presentada en el I Congreso de Universidades de Gaceta Universitaria celebrado en Salamanca 27-29 de noviembre de 2002.

Mora, J.-G. (2003). Documento marco II Congreso de Universidades Gaceta Universitaria, La calidad de las universidades: un paso hacia el futuro. Universidad de Santiago de Compostela, 10-12 de noviembre de 2003.

Mora, J. G. y Vieira, M. J. (2005). Los procesos de acreditación en el desarrollo de las universidades: visión europea comparada. En CINDA, Los procesos de acreditación en el desarrollo de las universidades (pp. 119-133). Santiago de Chile: CINDA

Mosier, R. E., \& Schwarzmueller, G. J. (2002). Benchmarking in Student Affairs. En B. E. Bender, \& J. H. Schuh (Eds.), Using Benchmarking to Inform Practice in Higher Education: New Directions for Higher Education (pp. 103-112). Jossey-Bass.

Neave, G., \& Van Vught, F. A. (1994). Prometeo encadenado. Estado y educación superior en Europa. Barcelona: Editorial Gedisa. 
OECD. (2005). Education at a Glance. Paris: Organisation for Economic Co-operation and Development.

Pardee, C. F. (2000). Organizational models for academic advising. En V. N. Gordon, \& W. R. Habley (Eds.), Academic Advising. A comprehensive handbook (pp. 192-209). San Francisco: Jossey-Bass.

Ponz, F. (1996). Espíritu universitario. En V. García Hoz (Dir.), La educación personalizada en la universidad (pp. 81-130). Madrid: Ediciones Rialp.

Porta, J. (1998). Arquetipos de universidades: de la transmisión de los saberes a la institución multifuncional. En J. Porta y M. Lladonosa (Eds.), La Universidad en el cambio de siglo (pp. 29-63). Madrid: Alianza Editorial.

Pozo Muñoz, C. (2000). El fracaso académico en la universidad: evaluación e intervención preventiva. Almería: Servicio de Publicaciones de la Universidad de Almería.

Quintanilla, M. A. (1998). El reto de la calidad en las universidades. En J. Porta y M. Lladonosa (Eds.), La Universidad en el cambio de siglo (79-103). Madrid: Alianza.

Reichert, S., \& Tauch, C. (2003). Trends III. Progress towards the European Higher Education Area. Bruselas: European University Association.

Reichert, S., \& Tauch, C. (2005). Trends IV European Universities. Implementing Bologna. Bruselas: European University Association.

Rey, A. A. (1998). Planificación estratégica, gestión de la calidad total y sistemas de información en la universidad. Planificación Estratégica y Mejora de la Calidad en la Universidad, Universidad del País Vasco, 2-4 diciembre de 1998.

Rodríguez Espinar, S. (2004). Manual de tutoría universitaria. Recursos para la acción. Barcelona: Ediciones Octaedro.

Rodríguez Espinar, S. et al. (1993). Teoría y práctica de la orientación educativa. Barcelona: PPU.

Rodríguez Moreno, M. L. (1995). Orientación e intervención psicopedagógica. Barcelona: CEAC.

Rodríguez Moreno, M. L. (2002). Hacia una nueva orientación universitaria. Barcelona: Edicions Universitat de Barcelona.

Sánchez García, M. F. (1998). Análisis y valoración de necesidades y servicios de orientación en las universidades de la Comunidad de Madrid. Tesis doctoral, Madrid: UNED.

Sandeen, A. (2001). Organization, functions, and standards of practice. En S. R. Komives, \& D. B. Woodward (Eds.), Student Services: A Handbook for the Profession (pp. 435-457). San Francisco: Jossey-Bass.

Sanz García, N. (2001). Orientación universitaria: asesoramiento académico personal. Premios nacionales de investigación educativa 2000 (pp. 103-127). Madrid: Secretaría General Técnica.

Sanz Oro, R. (2001). Orientación psicopedagógica y calidad educativa. Madrid: Pirámide.

Saulnier-Cazals, J. (1997). Educación en la orientación en la universidad. En P. Apodaca y C. Lobato (Eds.), Calidad en la Universidad: Orientación y Evaluación. (pp. 102-111). Barcelona: Editorial Laertes.

Sebkova, H. (2002). Accreditation and Quality Assurance in Europe. Higher Education in Europe, 27 (3), 239-247.

Sporn, B. (2003). Trends relating to higher education reform in Europe: an overview. En H. Eggings (Ed.), Globalization and Reform in Higher Education (pp. 117-129). England: Open University Press

Taylor, W. (2003). Steering change in tertiary education. En H. Eggings (Ed.), Globalization and Reform in Higher Education (pp. 11-31). England: Open University Press. 
Teichler, U. (1999). Higher education policy and the world of work: changing conditions and challenges. Higher Education Policy, 12 (4), 285-312.

Terenzini, P. T., Pascarella, E. T., \& Blimling, G. S. (1999). Students' out-of-class experiences and their influence on learning and cognitive development: a literature review. Journal of college student development, 40 (5), 610-623.

Thelin, J. R. (2001). Historical overview of American Higher Education. En S. R. Komives, \& D. B. Woodard (Eds.), Student Services: A Handbook for the Profession (pp. 3-21). San Francisco, CA: Jossey-Bass.

UNESCO. (1998). World Declaration on Higher Education for the Twenty-first Century: Vision and Action. Documento de la World Conference on Higher Education: Higher Education in the Twenty-First Century: Vision and Action, Paris, 5-9 octubre de 1998.

Vélaz de Medrano, C. (1998). Orientación e intervención psicopedagógica. Concepto, modelos, programas y evaluación. Málaga: Aljibe.

Vidal, J., Díez, G., \& Vieira, M. J. (2003). Guidance services in Spanish universities. Tertiary Education and Management, 9 (4), 267-280.

Watts, A. G., \& Van Esbroeck, R. (1998a). New Skills for New Futures: Higher Education Guidance and Counselling Services in the European Union. Brussels: VUB University Press. VUBPRESSFEDORA.

Watts, A. G., \& Van Esbroeck, R. (1998b). New skills for a holistic careers guidance model. The International Careers Journal (on-line), June, Available: www.careers-cafe.com.

Watts, A. G., \& Van Esbroeck, R. (2000). New skills for new futures: a comparative review of higher education guidance and counselling services in the European Union. International Journal for the Advancement of Counselling, 22 (3), 173-187.

Fecha de recepción: 05-02-05

Fecha de revisión: 17-01-06

Fecha de aceptación: 25-04-06 